\title{
Reproduction in vitro des effets de la pollution par le dioxyde de soufre sur quelques lichens
}

\author{
Max BEDENEAU \\ I.N.R.A., Station de Recherche sur la Forêt et l'Environnement \\ Centre de Recherches forestières d'Orléans \\ Ardon, F 45160 Olivet
}

\begin{abstract}
Résumé
Trois espèces de lichens, Lecanora conizaeö̈des (Nyl), Parmelia physodes (L) et Parmelia caperata (L.) Ach, ont été exposées à des flux constants de $\mathrm{SO}_{2}$ en atmosphère sèche et saturée.

Pour ces expériences, un appareil original a été conçu. Ses différents composants sont décrits et discutés.

Les dégâts causés par le $\mathrm{SO}_{2}$ lors de ces expériences sont semblables à ceux observés sur le terrain, un massif forestier soumis à pollution.
\end{abstract}

Les effets de la pollution atmosphérique sur la végétation font l'objet de nombreux travaux. L'attention des chercheurs a été attirée par les lichens du fait de leur sensibilité aux polluants atmosphériques : les espèces les plus sensibles disparaissent au fur et à mesure que la pollution s'accroît. Cette particularité de ces végétaux permet de tracer des cartes de pollution atmosphérique sur l'ensemble d'une région.

Cependant, les effets que l'on peut attribuer à un polluant déterminé sont encore peu connus.

Dans une précédente étude, nous avons fait mention de la dégradation de différentes espèces lichéniques dans un massif forestier soumis à pollution. Afin de reproduire les dégâts observés sur le terrain, nous avons construit une installation de pollution artificielle, dans laquelle nous avons exposé les espèces rencontrées en forêt, mais provenant de zones exemptes de pollution, à un flux constant de $\mathrm{SO}_{2}$.

La présente étude décrit la conception de cet appareil de pollution artificielle et les expérimentations sur les espèces lichéniques retenues. 


\section{I. - Matériel et méthodes}

Les expériences de pollution artificielle peuvent se classer ainsi :

- Fumigation dans la nature :

- Sans contrôle des doses émises (SKYE, 1968).

- Avec contrôle des doses émises (DE CoRmIs, 1977).

- Fumigation en laboratoire :

- Polluant en atmosphère confinée : les végétaux sont placés dans une enceinte close, à atmosphère de teneur donnée de polluant (RAO \& LEBlanC, 1965).

- Courant gazeux continu (Goosens, 1976).

- Immersion dans une solution du polluant considéré (TürK \& WIRTH, 1975).

L'installation de pollution artificielle que nous avons construite est basée sur la méthode du courant gazeux continu : un flux de gaz, de vitesse et de concentration constantes, inonde des thalles de lichens.

Les appareils déjà existants possèdent quelques contraintes importantes :

- Le gaz utilisé est un gaz fabriqué spécialement par l'industrie, d'un prix de revient élevé.

- Des mécanismes de régulation et d'analyse du polluant asservissent ces installations à une source d'énergie (l'électricité), ce qui rend ces appareils fixes.

- Les enceintes où sont placés les éléments à expérimenter sont conçues pour un sujet précis, et peu propices à l'accueil d'autres matériels.

Il nous a semblé intéressant de concevoir un appareil simple, pouvant accepter plusieurs gaz polluants de façon à tester l'effet seul ou combiné de ces gaz.

Les enceintes doivent être facilement transformables pour accepter n'importe quel type de sujet à expérimenter.

Le fait que cet appareil soit mobile peut permettre, grâce à un circuit témoin, d'étudier l'effet des gaz polluants in situ sur des échantillons de plantes.

Nous avons été amené à choisir la formule suivante (figure 1).

Soit deux bouteilles d'acier allégé, une contenant de l'air pur, l'autre contenant un mélange dosé de $\mathrm{SO}_{2}$ et d'air, reliées chacune à un débitmètre.

De ce débitmètre partent deux tuyaux reliés chacun à l'avant de deux enceintes. Dans l'une, on dispose une coupelle remplie d'eau distillée avec du papier filtre, de façon à avoir un taux d'hygrométrie de 100 p. 100 . Le fait d'avoir des enceintes humides se justifie par les études de TüRK et al. (1974) qui ont démontré l'action plus nocive du $\mathrm{SO}_{2}$ en atmosphère humide.

L'autre enceinte n'a aucun dispositif de ce genre (enceinte sèche). Chaque enceinte est close de façon étanche au moyen de mastic synthétique. La plasticité de ce matériau en permet l'ouverture périodique. A l'arrière des deux enceintes, il y a un orifice de sortie des gaz relié de façon étanche à un flacon contenant de l'acétate de zinc en solution, destiné à piéger le $\mathrm{SO}_{2}$ (MALBosc, 1967). 


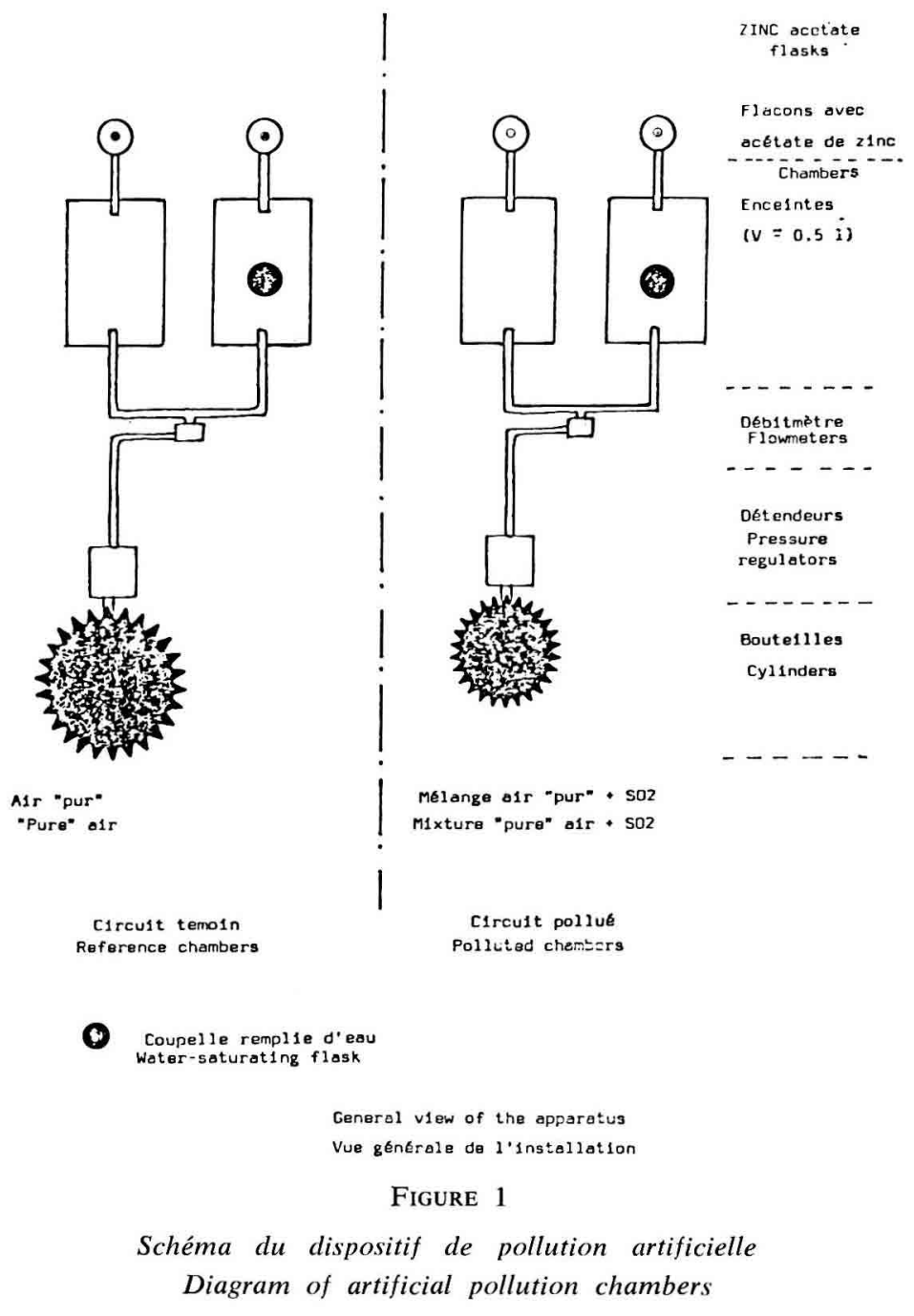

On obtient donc deux circuits, un témoin (air pur), un pollué, reliés chacun à deux enceintes, une humide, l'autre sèche.

En détendant l'air contenu dans les bouteilles, on crée un flux de gaz à l'intérieur des enceintes, flux que l'on règle par la pression de sortie de la bouteille, puis par le débitmètre.

La bouteille du circuit témoin est remplie d'air, préalablement filtré (sans poussières, graisses et eau), au moyen d'un compresseur. La pression est de 180 bars. 
Généralement, la production et le contrôle de $\mathrm{SO}_{2}$ nécessitent un appareillage complexe. C'est pourquoi, recherchant une voie peu onéreuse, nous avons choisi de produire en laboratoire une quantité connue de $\mathrm{SO}_{2}$ que l'on transvase dans une bouteille vide. On rajoute ensuite la quantité nécessaire d'air pur (à l'aide d'une autre bouteille préalablement remplie), pour obtenir la concentration voulue.

\subsection{Le gaz}

\subsection{Choix des divers paramètres}

Pour produire le $\mathrm{SO}_{2}$, nous avons utilisé la réaction de l'acide sulfurique sur le sodium sulfite selon la formule :

$$
\mathrm{H}_{2} \mathrm{SO}_{4}+\mathrm{Na}_{2} \mathrm{SO}_{3} \rightarrow \mathrm{Na}_{2} \mathrm{SO}_{4}+\mathrm{H}_{2} \mathrm{O}+\mathrm{SO}_{2}
$$

Braun (1976) a utilisé la même réaction lors d'expériences sur les épicéas et nous nous sommes largement inspiré de ses travaux pour les temps de réaction et les doses de $\mathrm{Na}_{2} \mathrm{SO}_{3}$.

La quantité de $\mathrm{SO}_{2}$ dégagée, $z$, lors de cette réaction va être telle que :

$$
\mathrm{z}=\mathrm{x} \cdot\left(\mathrm{Na}_{2} \mathrm{SO}_{3}\right) \cdot \frac{126}{64}\left(\text { poids atomique } \frac{\mathrm{Na}_{2} \mathrm{SO}_{3}}{\mathrm{SO}_{2}}\right)
$$

où $x$ est la quantité de $\mathrm{Na}_{2} \mathrm{SO}_{3}$. On tire $\mathrm{x}=\mathrm{z} \cdot \frac{64}{126}$

Cette quantité $\mathrm{z}$ va se dégager dans un certain volume v. Si on ouvre un autre volume $\mathrm{v}_{1}$, la quantité de $\mathrm{SO}_{2}$ migrant dans ce volume, $\mathrm{z}_{1}$, va être telle que :

$$
\frac{\mathrm{z}}{\mathrm{z}_{1}}=\frac{\mathrm{v}}{\mathrm{v}_{1}} . \text { On tire } \mathrm{z}=\frac{\mathrm{v}}{\mathrm{v}_{1}} \cdot \mathrm{z}_{1}
$$

En associant (1) et (2), on obtient :

$$
\mathrm{Q}\left(\mathrm{Na}_{2}, \mathrm{SO}_{3}\right)=\mathrm{z}_{1} \cdot \frac{126}{64} \cdot \frac{\mathrm{v}}{\mathrm{v}_{1}}
$$

La quantité finale de $\mathrm{SO}_{2}$ à obtenir est calculée ainsi :

(1) Concentration désirée en $\mathrm{ppm}\left({ }^{*}\right) \times 2857=\mathrm{Q}\left(\mathrm{SO}_{2}\right)$ en $\mathrm{mg}$.

(2) $\mathrm{Q}\left(\mathrm{SO}_{2}\right) \times($ volume de la bouteille $\times$ pression $) / 1000$.

La quantité de $\mathrm{Na}_{2} \mathrm{SO}_{3}$ nécessaire est obtenue en multipliant $\mathrm{Q}$ (SO.2) par les facteurs décrits précédemment.

$\left(^{*}\right)$ ppm $=1$ partie de $\mathrm{SO}_{2}$ pour $10^{\circ}$ parties d'air. $1 \mathrm{ppm}=2857 \mu \mathrm{g} / \mathrm{m}^{3}$ dans les conditions normales de température et de pression. 
Le $\mathrm{SO}_{2}$ ainsi fabriqué est transvasé dans une bouteille de 15 litres dans laquelle on a préalablement fait le vide $(74 \mathrm{~mm} \mathrm{Hg})$.

On rajoute ensuite de l'air comprimé provenant d'une bouteille de 50 litres remplie à 200 bars. Le transvasement s'effectue jusqu'à équilibre de pression entre les deux bouteilles.

A toutes les étapes de la production, on contrôle la teneur en $\mathrm{SO}_{2}$ à laide de tubes colorimétriques (DraEgER, 1976), couramment utilisés par les hygiénistes.

Pour la vérification dans le flacon du laboratoire, on utilise des tubes permettant une détection de 1 à $20 \mathrm{ppm}$. Lors du transvasement, on vérifie à différentes pressions, à l'aide de tubes allant de 0,1 à 3 ppm.

Le gaz envoyé dans le circuit témoin est de l'air pompé dans le milieu ambiant et préalablement filtré et desséché, compressé à 200 bars dans une bouteille de 50 litres.

\subsection{Dose}

GilberT (1969) a démontré que l'absorption du soufre par les lichens était un phénomène actif.

O'Hare \& Williams (1973) ont analysé les thalles qu'ils avaient fumigés à des concentrations comprises entre 0,1 et $0,2 \mathrm{ppm}$; les valeurs maximales sont de l'ordre de $3500 \mathrm{ppm}$.

Le problème est donc de se situer à des doses et des débits tels que les lichens exposés ne puissent tout fixer.

Une dose de $10000 \mu \mathrm{g}$ de $\mathrm{SO}_{2}$ par gramme de lichen et par mois correspond à $13,89 \mu \mathrm{g} / \mathrm{g} /$ heure. Afin de pouvoir observer facilement les dégâts occasionnés par le $\mathrm{SO}_{2}$, nous avons choisi une concentration de $0,5 \mathrm{ppm}$, soit $1429 \mu \mathrm{g} / \mathrm{m}^{3}$. Cette dose n'est pas irréaliste, puisque de telles concentrations ont été observées à Roumare pendant 12 heures (Service des Mines, 1980).

\subsection{Débit}

Certains auteurs (DiBBEN, 1971) ont constaté des altérations d'espèces lichéniques placées en conditions artificielles, dues par exemple à des envahissements par des champignons lichénicoles non lichénisés ou des algues.

Il s'est effectivement développé, à des flux très faibles $(<51 / \mathrm{h})$ des champignons parasites dans les enceintes humides. Aucune colonisation de ce genre n'a eu lieu dans les enceintes sèches. C'est en fonction de ces données que nous avons choisi un flux de $81 / \mathrm{h}$ et une hygrométrie de $100 \mathrm{p}$. 100 dans les enceintes humides. Il semble que les champignons parasites et les algues soient balayés par ce flux et ne puissent se développer, bien qu'un examen microscopique relève la présence d'algue Pleurococcus se développant dans les anfractuosités du cortex supérieur des espèces exposées. 
Le débit a été calculé :

$$
13,89 \mu / \mathrm{g} / \text { heure } \times \frac{1}{1429} \mathrm{~m}^{3}\left({ }^{*}\right)=9,72 \quad 1 / \text { heure }
$$

Les conditions expérimentales (poids de lichen, renouvellement des bouteilles) nous ont obligé à réduire ce débit et à adopter finalement un débit de $8 \mathrm{t} / \mathrm{heure}$ qui, vu le volume de l'enceinte $(0,5 \mathrm{l})$, permet un renouvellement de 16 fois par heure, chiffre adopté par Goosens (1976).

\subsection{Durée}

Les durées d'exposition varient, selon les auteurs, de quelques heures à des doses très élevées à plusiers jours à doses moyennes. Lors des transplantations dans la nature, ces expositions durent même plusieurs mois (FERRY \& CoppINs, 1979).

Nous avons choisi de fumiger les lichens jusqu’à ce que des dégâts visibles apparaissent dans l'une quelconque des enceintes. Nous avons repris le protocole de O'Hare \& Williams (1975) pour l'estimation des dégâts.

Dans la pratique, ceci nous a conduit à fumiger pendant trois semaines consécutives le lichen choisi, durée proche de celle de WIRTH \& TürR (1975) et GooSENS (1976).

\subsection{Température}

Aucun dispositif de régulation de température n'a été prévu à l'intérieur des enceintes. L'ensemble de l'installation est placé dans une pièce d'entrepôt, chauffée par un radiateur électrique. Les expérimentations ont eu lieu durant les mois d'hiver et de printemps, la température mesurée au niveau des enceintes a toujours été comprise entre $15^{\circ} \mathrm{C}$ et $21^{\circ} \mathrm{C}$.

\subsection{Eclairement}

Nous avons installé un dispositif d'éclairage composé de 10 tubes néons montés en série (MAZDAFLUOR - TF 40/BBL).

Afin de déterminer la distance à laquelle nous allions placer cette rampe lumineuse, nous avons mesuré l'éclairement dans un sous-bois de chênes très clair, où croissaient particulièrement bien des thalles de Parmelia caperata.

Nous avons trouvé des valeurs de l'ordre de 5000 lux, ceci au mois de janvier par temps clair (voir figure 2).

Nous avons placé la rampe lumineuse à 65 centimètres des enceintes, ce qui donne un éclairement d'environ 4600 lux, compte tenu de la légère absorption par le plastique.

$\left(^{*}\right)$ Si $1 \mathrm{~m}^{3}$ contient $1429 \mu \mathrm{g}$ de $\mathrm{SO}_{2}, 1 \mu \mathrm{g}$ de $\mathrm{SO}_{2}$ est contenu dans $\frac{1}{1429} \mathrm{~m}^{3}$. 


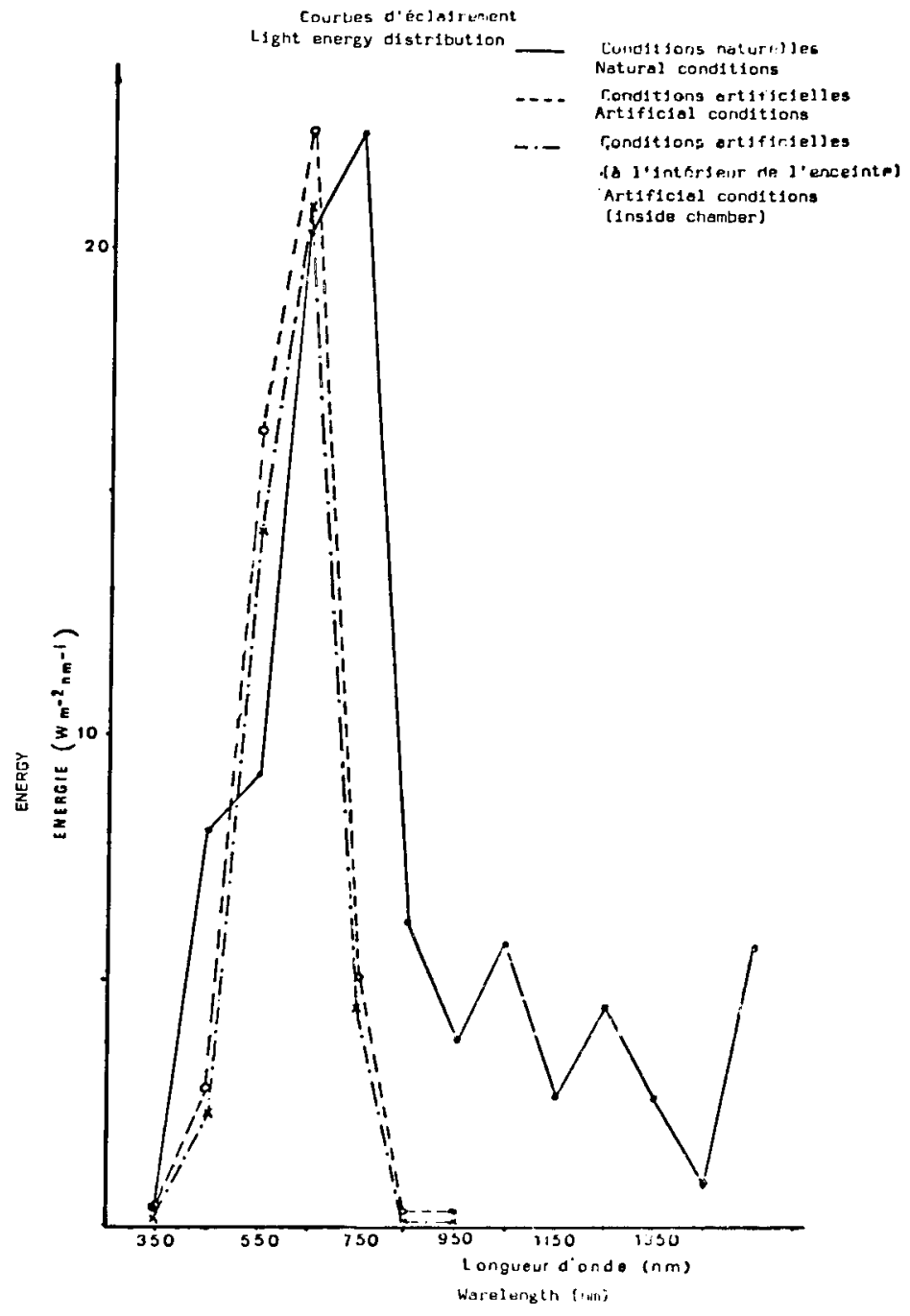

Figure 2

Courbes d'éclairement

Light energy distribution

Le photopériodisme, réglé par une horloge électrique, est de 12 heures.

Ces données nous permetent de placer les lichens dans des conditions proches des paramètres naturels (Clauzade, 1970) (voir photo). 


\subsection{Choix des espèces lichéniques}

Nous désirons reproduire en atmosphère confinée des conditions de pollution par $\mathrm{SO}_{2}$ pour observer la dégradation des lichens. Nous avons choisi trois espèces, reconnues de sensibilité différente au $\mathrm{SO}_{2}$ (HAwksworth \& Rose, 1970). Il s'agit de :

- Lecanora conizaeoüdes Nyl. : espèce résistant à la pollution.

- Parmelia physodes (L.) Ach. : espèce moyennement résistante.

- Parmelia caperata (L.) Ach. : espèce sensible.

Des thalles vigoureux de ces différentes espèces ont été prélevés dans des zones non polluées (forêt de Lyons, environs d'Orléans) et exposés dans les enceintes avec l'écorce qui les supporte.

\subsection{Exposition}

Quatre échantillons d'une même espèce, provenant du même prélèvement, sont placés dans chacune des enceintes correspondant pour Lecanora conizaeoïdes et Parmelia physodes à :

— pollué sec : 0,5 ppm SO $\mathrm{SO}_{2}, \quad 0$ p. 100 hygrométrie,

— pollué humide : $0,5 \mathrm{ppm} \mathrm{SO}_{2}, 100$ p. 100 hygrométrie,

— témoin sec : $0,0 \mathrm{ppm} \mathrm{SO}_{2}, \quad 0$ p. 100 hygrométrie,

- témoin humide : $0,0 \mathrm{ppm} \mathrm{SO}_{2}, 100$ p. 100 hygrométrie.

Les mêmes répétitions ont lieu pour les thalles de Parmelia caperata, mais à 0,25 ppm de $\mathrm{SO}_{2}$.

\section{II. - Résultats}

\subsection{Morphologie}

Sur toutes les espèces exposées, nous avons constaté des changements de couleur du thalle sur les échantillons placés dans les enceintes polluées. Ces décolorations sont plus importantes sur les sujets placés en enceintes humides.

Sur $P$. caperata, les premières décolorations (inférieures à 25 p. 100 de la surface du thalle) apparaissent au bout d'une semaine. Elles sont nettement situées au centre du thalle, c'est-à-dire dans la partie la plus âgée.

Après deux semaines, le thalle présente un blanchissement plus intense (entre 25 et 50 p. 100 de la surface du thalle), s'étendant sur les lobes marginaux. Quelques points blanchâtres apparaissent sur les thalles des circuits témoins, mais ils ne s'étendent pas à l'ensemble du thalle.

Les décolorations des thalles pollués, plus particulièrement en atmosphère humide, s'étendent sur 50 p. 100 de la surface du thalle en fin d'expérience. 
P. physodes, pollué à $0,5 \mathrm{ppm}$ de $\mathrm{SO}_{2}$, connaît au bout d'une semaine des plages blanchâtres ( $<25$ p. 100 de la surface du thalle) situées au centre du thalle. Ces plages s'étendent sur les lobes marginaux la semaine suivante, puis à l'ensemble du thalle. Le sujet placé en atmosphère sèche polluée est nettement moins décoloré, mais présente une pruine sur l'ensemble du thalle. Les sujets des enceintes témoins subissent, comme les thalles de $P$. caperata quelques décolorations blanchâtres.

Sur Lecanora conizaeoïdes, pollué à $0,5 \mathrm{ppm}$ de $\mathrm{SO}_{2}$, on voit d'abord une turgescence des apothécies qui deviennent brunâtres au bout d'une semaine, dans l'enceinte polluée humide. Le sujet placé dans cette enceinte voit son thalle fractionné en petits paquets et bruni sur 25 p. 100 de sa surface. Le sujet de l'enceinte sèche ne subit pas ce fractionnement, mais les apothécies sont intensément brunes. Après 3 semaines, les thalles situés en atmosphère humide sont décolorés en plages brunes et blanches, les thalles des enceintes témoins ne subissent aucune dégradation, seules quelques apothécies du sujet placé en atmosphère humide deviennent brunes.

\subsection{Anatomie}

Sur toutes les espèces exposées en enceintes polluées, nous avons pu constater des altérations de lalgue symbiotique.

Dans les sujets prélevés avant l'expérience, on peut observer des soralies $\left(^{*}\right)$. On ne peut en voir sur les sujets exposés, quel que soit le circuit (pollué ou témoin). Il semblerait que le flux gazeux balaye les sorédies (*), les dispersant dans les flacons situés en aval des enceintes.

La séquence de dégradation des espèces placées en atmosphère polluée humide est la suivante :

- dégradation de quelques cellules algales, avec d'abord une rétraction des chloroplastes ;

- dégradation de l'ensemble des cellules algales, décoloration complète des cellules mortes, cytoplasme granuleux des cellules subsistantes;

- migration de ces cellules à l'intérieur de la médulle, où elles forment avec les hyphes mycéliennes des glomérules présentant l'aspect des sorédies;

- ces cellules meurent à leur tour, les hyphes mycéliennes sont plus nombreuses autour des algues.

Durant ces transformations, on observe une coloration différente du cortex supérieur, ainsi que son épaississement. En maints endroits, il se fractionne. Des cellules de Pleurococcus se développent en chaîne dans ces crevasses, pouvant même atteindre la médulle $\left(^{* *}\right.$ ) (il est possible de faire la différence entre la Chaetophoracée Pleurococcus sp. et l'algue symbiotique, dans notre cas une Chlorococaccée du genre Trebouxia).

(*) Sorédies; soralies : les sorédies sont de fines granulations de 25 à 100 microns de diamètre, formées par quelques cellules algales entremêlées et entourées de filaments mycéliens. Elles prennent naissance en groupes appelées soralies. Iâches.

$\left(^{* *}\right)$ Médulle : «moelle», partie la plus interne du lichen, composée d'hyphes mycéliennes 


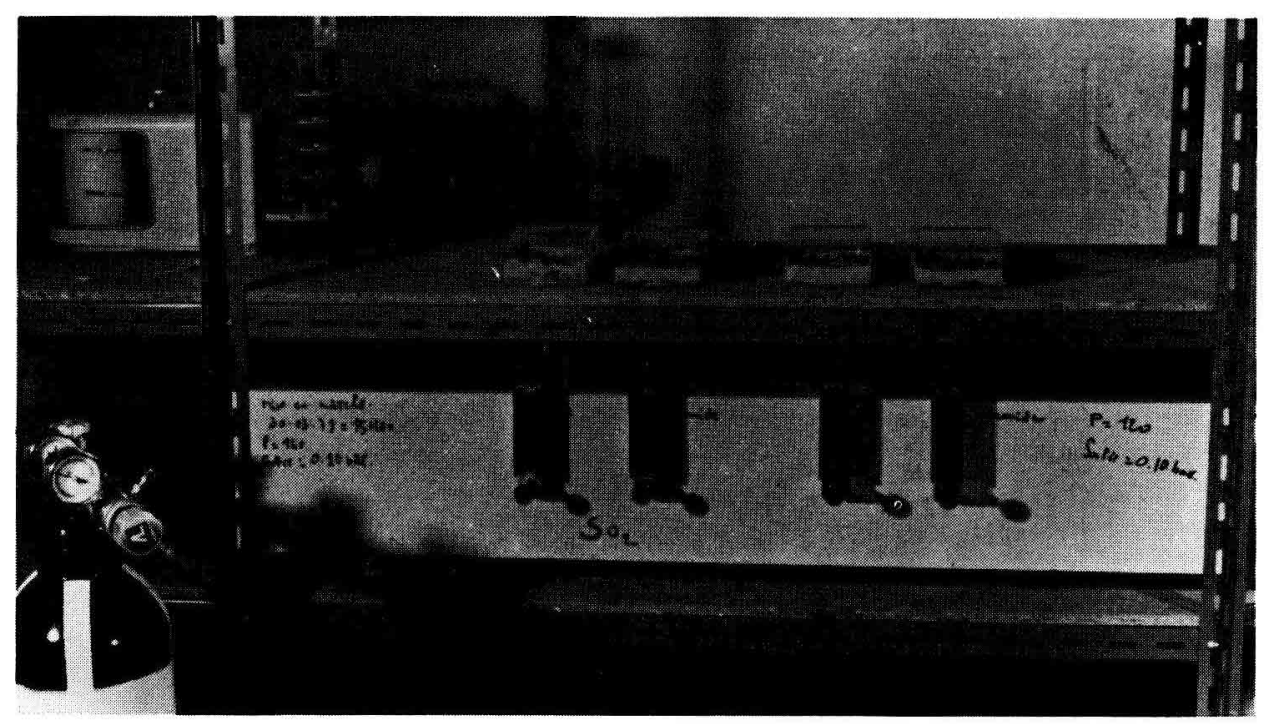

Légende : Vue générale de l'installation

General view of the apparatus

Dans les circuits pollués secs, on observe une mortalité importante des cellules algales, sans que celles-ci migrent vers la médulle. On observe le même phénomène dans le circuit témoin sec. Le cortex supérieur des espèces placées dans ces enceintes présente de nombreux fractionnements sans que se développe l'algue Pleurococcus.

On n'observe aucune variation notable dans le circuit témoin humide.

Selon les espèces exposées, le nombre de cellules algales mortes varie. Ainsi, dès la première semaine, environ le quart des cellules est touché chez $P$. caperata (à $0,25 \mathrm{ppm}$ de $\mathrm{SO}_{2}$ ), la même proportion chez $P$. physodes (à $0,5 \mathrm{ppm}$ ), alors que seulement 10 p. 100 présentent des altérations, principalement celles situées sur le rebord thallin des apothécies, sur $L$. conizaeoïdes. Vers la fin des expériences, toutes les cellules, chez toutes les espèces, présentent des altérations notables.

\subsection{Substances lichéniques}

Nous avons suivi tout au long de l'expérience sur chacune des espèces, les substances lichéniques $\left({ }^{*}\right)$.

(*) Nous avons utilisé la méthode de chromatographie sur couches minces décrite par Culberson (1970) : trois quantités égales d'extrait acétonique de lichen sont déposées sur trois plaques chromatographiques, ainsi que des extraits de Parmelia acetabulum, qui contient deux substances témoins, l'atranorine et l'acide norstictique. Ces plaques sont développées dans trois solvants différents, A, B et $\mathrm{C}$.

La migration des substances lichéniques est différente selon les solvants, chaque tache est repérée par rapport aux lignes de migration de l'atranorine et de l'acide norstictique. Pour chacun des bains, chaque tache est affectée d'une classe : inférieure à la ligne de l'atranorine, égale ou supérieure, etc. Selon les différentes classes obtenues, un système de cartes perforées et d'aiguilles permet l'identification de la substance, si celle-ci a été décrite. 
Dans une étude précédente (Bedeneau, 1980), sur l'évolution des substances lichéniques en milieu pollué, il avait été mis en évidence, sur les extraits de Parmelia physodes, la présence puis la disparition d'une tache rouge, migrant à un rF de 0,25 dans le solvant C. Cette substance, non décrite par CuLBERSON (1969-1970), apparaît dans les zones moyennement polluées $\left(\simeq 60 \mathrm{lg} \mathrm{SO}_{2} / \mathrm{m}^{3}\right.$ - LEROND, 1978), puis disparaît dans les sites nouvellement pollués ou récemment colonisés par Parmelia physodes.

Dans la présente expérience, on observe en deuxième semaine, sur la chromatographie d'extrait de Parmelia physodes, cette tache rouge. Elle est encore présente en troisième semaine.

Comme Klee \& Steubing (1977), nous n'avons noté aucune variation de concentration de l'atranorine tant sur $P$. caperata que sur $P$. physodes.

Il n'apparaît aucune tache surnuméraire sur les chromatogrammes d'extrait de $L$. conizaeö̈des et il n'y a aucune variation notable de la teneur en acide fumarprotocétrarique.

\section{III. - Discussion}

Les altérations morphologiques des lichens exposés au $\mathrm{SO}_{2}$ sont semblables à celles observées sur le terrain, notamment :

- les décolorations des thalles,

- le fractionnement de ceux-ci en petits amas.

Comme TüRK et al. (1974), nous constatons que les dégâts sont plus importants en atmosphère humide qu'en atmosphère sèche. On peut formuler quelques hypothèses pour expliquer ce phénomène :

- Les composés soufrés sont plus nocifs en atmosphère humide du fait de leur combinaison avec l'eau (il en résulte $\mathrm{H}_{2} \mathrm{SO}_{4}$ par exemple).

- L'activité biologique des lichens, directement conditionnée par l'eau, est plus intense quand le milieu est saturé. De ce fait, il absorbe de façon plus importante les divers composants de l'atmosphère.

Il a été démontré que l'activité des lichens est plus importante en atmosphère humide (Clauzade, 1970). TürK et al. (1974) ont démontré la nocivité des composés soufrés, notamment par l'acidification du milieu. Pucketr et al. (1973) précisent que toutes les formes de $\mathrm{SO}_{2}$ en solution sont des oxydants d'autant plus importants que le $\mathrm{pH}$ est bas.

Il semble donc que ce soit la conjonction de deux phénomènes (taux d'hygrométrie, présence de $\mathrm{SO}_{2}$ ) qui entraîne la dégénérescence des lichens en milieu pollué.

Par cette expérimentation, nous avons reproduit les dégâts anatomiques, déjà décrits dans la littérature, notamment par RIEUX (1977). Nous retrouvons effectivement des altérations de l'algue symbiotique, tuée au bout de quelques semaines. Le comportement saprophytique du champignon pose un problème, de même que l'appa- 
rition d'une substance lichénique nouvelle (qui, comme toute substance lichénique, est sécrétée par les hyphes mycéliennes).

Des recherches ultérieures sont nécessaires car plusieurs questions se posent :

Est-ce la mort de quelques algues, est-ce la rupture d'un équilibre qui provoque ce changement de comportement?

Il nous semble nécessaire d'apporter quelques améliorations à l'appareil de pollution artificielle que nous avons conçu :

- En atmosphère sèche ( 0 p. 100 d'hygrométrie), l'activité biologique des lichens est extrêmement ralentie.

- Par contre, en atmosphère humide, à éclairement constant, cette activité est au contraire fortement stimulée.

Dans la nature, ces activités sont intermittentes, liées à la luminosité et à l'économie de l'eau.

Une première amélioration de notre appareil consisterait donc à réguler le taux d'hygrométrie (par exemple à l'aide de solution de potasse). La mesure de l'activité photosynthétique pourrait permettre de définir les intervalles de variation optimum du taux d'hygrométrie, de suivre plus précisément les altérations en atmosphère polluée.

Les doses de polluants employées sont exagérément élevées pour des périodes de temps aussi longues ( 3 semaines). N'importe quel végétal subit des altérations notables à de tels niveaux de pollution.

Néanmoins, il est troublant de reconstituer, à des doses si fortes, les dégâts observés sur le terrain. Il reste donc à déterminer les seuils de sensibilité des lichens au $\mathrm{SO}_{2}$ :

- Ces végétaux sont-ils détruits par des pointes de pollution (de l'ordre de $2000 \mu \mathrm{g} / \mathrm{m}^{3}$ pendant 4 heures) ?

- Ou sont-ils affaiblis, puis détruits par un enchaînement de phénomènes complexes (parasitisme des algues et des champignons lichénicoles), par une pollution à un taux beaucoup plus faible (de l'ordre de $100 \mu \mathrm{g} / \mathrm{m}^{3}$ pendant un mois) ?

\section{IV. - Conclusion}

A l'aide d'un dispositif simple, il a été possible de reproduire les dégâts macroscopiques observés sur le terrain (BEDENEAU, 1980).

La plasticité de chacun des éléments de l'installation permet l'adaptation rapide, soit à différents types de matériels à tester, soit à différents polluants.

Ainsi l'installation est utilisée pour déterminer la sensibilité de microarthropodes du sol à la pollution soufrée (MORVAN, 1981, en cours). 
Une étude se poursuit sur les lichens en prenant comme polluant le fluor sous forme gazeuse. Il est envisagé d'étudier prochainement le mélange $\mathrm{SO}_{2}$-fluor sur ces végétaux.

Enfin, le dernier «étage», filtration, peut être aisément remplacé par un étage d'analyse : photosynthèse, teneur en polluant, permettant ainsi une approche physiologique du phénomène de dégradation des plantes ou des animaux par les polluants atmosphériques.

Reçu pour publication le 7 juillet 1981

\section{Summary}

Artificial $\mathrm{SO}_{2}$ effects on lichens

The effects of $\mathrm{SO}_{2}$ were tested on three species of lichen in dry and water saturated atmospheres : on Lecanora conizaeoïdes ( $\mathrm{Nyl})$, Parmelia physodes (L), and Parmelia caperata (L) Ach.

For these experiments an original fumigation apparatus was designed. Its various components are described and discussed here.

The effects observed in the lichens are similar to those observed in the field, in a polluted forest.

\section{Références bibliographiques}

BEDENEAU M., 1980. Evolution des caractères morphologiques, anatomiques et biochimiques de quelques lichens subsistant en milieu forestier pollué. Thèse, Université d'Orléans.

BraUn G., 1976. Eine einfache Methode zur SO.. Begasung von Fichtenpfropflingen in Labor. Eur. J. For. Pathol., 6, 329-335.

Clauzade G., 1970. Les lichens et le milieu. In "Les lichens, étude biologique et flore illustrée ». P. Ozenda et G. Clauzade, Masson et Cie Ed., Paris, 97-109.

Comeau G., Leblanc F., 1972. Influence du Fluor sur le Funaria hygrometrica et l'Hypogymnia Physodes. Can. J. Bot., 50, 847-856.

De Cormis L., 1977. Dix années d'études des répercussions de la pollution de l'air sur les végétaux. I.N.R.A., Laboratoire d'étude de la Pollution atmosphérique.

Culberson C.F., 1969. Chemical and botanical guide to lichen products.

Culberson C.F., 1970. A standardized method for the identification of lichen products. J. Chromatogr., 46, 85-93.

DibBen M.J., 1971. Whole lichen culture in a phytotron. Lichenologist, 5, 1-10.

Draeger, 1976. Livre de poche concernant les tubes réactifs (texte établi par K. Leichnitz), $3^{\text {e }}$ édition. Draeger-Brandt S.A., 3, route de la Fédération, 67100 Strasbourg.

FERrY B.W, CoppINS B.J., 1979. Lichen transplant experiments and air pollution studies. Lichenologist, 11 (1), 63-73.

Gilbert O.L., 1969. Les effets du $\mathrm{SO}_{2}$ sur les lichens et les bryophytes autour Newcastle Upon Tyne. In Air Pollution Proceed Wageningen, 223-235. 
Goossens M., 1976. Réalisation de chambrettes pour la culture de cryptogames en atmosphère artificiellement polluée. Bull. Soc. r. Bot. Belg., 109, 49-54.

Hawksworth, Rose, 1970. Qualitative Scale for estimating Sulphur Dioxide Air Pollution in England and Wales using Epiphytic lichens. Nature, vol. 227, $\mathrm{n}^{\circ}$ 5 254, 145-148.

Klee R., Steubing L., 1977. Die Veränderung der Atranorinkonzentration in Laubflechten durch Licht, Wasserversorgung und $\mathrm{SO}_{2}$ Begasung. Bryologist, vol. 80, 171-174.

LEROND M., 1978. Courbes d'isopollution de la région de Rouen obtenues par l'observation des lichens épiphytes. Bull. Soc. Linn. Normandie, 106, 73-84.

Malbosc R., 1967. Contribution à l'étude de la détermination spécifique de l'anhydride sulfureux : polluant atmosphérique. Thèse, Université de Toulouse. Faculté mixte Médecine-Pharmacie, 1-91.

O'Hare G.P., Williams P., 1975. Some effects of sulphur dioxide flow on lichens. Lichenologist, 7, 116-120.

Puckett K.J., Nieboer E., Flora, Richardson, 1973. Sulphur dioxide : its effect on photosynthetic ${ }^{14} \mathrm{C}$ fixation in lichens and suggested mechanisms of phytotoxicity. New Phytol., 72, 141-154.

RaO D.N., Leblanc F., 1965. Effects of sulfur dioxide on the lichen alga with special reference to chlorophyll. Bryologist, 69, 69-75.

Rieux R., 1977. Végétation lichénique et pollution atmosphérique dans la zone de Fossur-Mer. Premières observations. Bull. Mus. Hist. nat. de Marseille, XXXVII, 93-107.

Service de l'Industrie et des Mines de Haute-Normandie, 1980. Rapport sur l'alerte du 18-1-1980, R.E.M.A.P.P.A., Dir. Interdépartementale de l'Industrie de Haute-Normandie, 11.

SKYE E., 1968. Lichens and air pollution (a study of cryptogamic epiphytes and environment in the Stockholm region). Acta phytogeogr. Suec., 52, 1-123.

Türk R., Wirth V., Lange O.L., 1974. $\mathrm{CO}_{2}$ Gaswechel-Untersuchungen zur $\mathrm{SO}_{2}$ Resistenz von Flechten. Oecologia (Berl.), 15, 33-64.

WIRTH V., TüRK R., 1975. Zur $\mathrm{SO}_{9}$ Resistenz von Flechten verschiedener Wormsform. Flora Bd, 164, S, 133-143. 